A. Boccuto, Department of Mathematics and Computer Science, via Vanvitelli 1, I-06123 Perugia, Italy. email:

boccuto@dipmat.unipg.it, boccuto@yahoo.it

D. Candeloro, Department of Mathematics and Computer Science, via

Vanvitelli 1, I-06123 Perugia, Italy. email: candelor@dipmat.unipg.it

\title{
SOBCZYK-HAMMER DECOMPOSITIONS AND CONVERGENCE THEOREMS FOR MEASURES WITH VALUES IN $l$-GROUPS
}

\begin{abstract}
We find a decomposition of the type of Sobczyk-Hammer for measures with values in l-groups, and also deduce some convergence theorems for such decompositions. Our procedure is based on some theorems of the type of Vitali-Hahn-Saks, and on the so-called Stone extension method.
\end{abstract}

\section{Introduction.}

In [3] and [4], we obtained some versions of the Lebesgue decomposition theorem, and of the Vitali-Hahn-Saks theorem for finitely additive measures with values in (super) Dedekind complete $l$-groups. In the quoted papers, the notion of convergence was related to $(D)$-sequences and therefore many of the concepts and proofs appear somewhat complicated. In this paper we investigate a different kind of decomposition, and require that the involved $l$-group is super Dedekind complete and weakly $\sigma$-distributive. This allows us to avoid the machinery of $(D)$-convergence [2], thanks to a powerful result concerning suitable subsequences of an $(O)$-sequence 2.7. Hence all the relevant convergence properties can be formulated and proved only by means of $(O)$-sequences, which are a more natural tool.

Key Words: l-groups, (RO)-convergence, uniform $s$-boundedness, uniform continuity, Vitali-Hahn-Saks theorems, Sobczyk-Hammer decomposition

Mathematical Reviews subject classification: 28B15, 28B05, 28A39, 42C10, 42C25, $46 \mathrm{G} 10$.

Received by the editors December 6, 2006

Communicated by: Stefan Schwabik 
The core of our research here concerns continuous and atomic l-groupvalued measures. We obtain sufficient conditions for continuity and uniform continuity. By means of these results, we deduce a decomposition of a measure, in the sense of Sobczyk-Hammer, that is into its continuous and atomic part. Finally, we give a convergence theorem for such decompositions.

In Section 2 we give the definitions and state some preliminary results about the techniques to be used later. In Section 3 we introduce and study continuity properties of measures, obtaining a sufficient condition for a sequence of finitely additive measures to be uniformly continuous, and also some useful relations between continuity and absolute continuity. Finally, in Section 4 we introduce the sectional decompositions, and, thanks also to the previous theorems, we find some results concerning existence and convergence for Sobczyk-Hammer decompositions.

\section{Preliminary Definitions and Results.}

We shall introduce now the main definitions we need, together with some results.

Definition 2.1. An Abelian group $(R,+)$ is called an l-group, if it is endowed with a compatible ordering $\leq$, and is a lattice with respect to it. An l-group $R$ is said to be Dedekind complete, if every nonempty subset of $R$, bounded from above, has supremum in $R$.

One important consequence of this definition is that convergence of series can be defined, at least when the terms are in $R_{0}^{+}=\{r \in R: r \geq 0\}$.

Definition 2.2. Given any sequence $\left(a_{n}\right)_{n}$ in $R_{0}^{+}$, we say that the series $\sum_{n=1}^{\infty} a_{n}$ is convergent if the set of all partial sums $\left\{s_{n}: n \in \mathbb{N}\right\}$ is bounded in $R$, where $s_{n}=\sum_{i=1}^{n} a_{i}$ for all $n$. If this is the case, we set $\sum_{n=1}^{\infty} a_{n}=\sup \left\{s_{n}\right.$ : $n \in \mathbb{N}\}$.

Convergence of series is also related to the so-called $(O)$-convergence, according with the following definition.

Definition 2.3. Given a sequence $\left(r_{n}\right)_{n}$ in $R$, we say that $\left(r_{n}\right)_{n}(O)$-converges to an element $r \in R$ if there exists a sequence $\left(p_{n}\right)_{n}$ in $R$, such that $p_{n} \downarrow 0$ (Such a sequence will be called an (O)-sequence.), satisfying $\left|r_{n}-r\right| \leq p_{n} \forall n \in \mathbb{N}$.

It is not difficult to see that a series $\sum_{n=1}^{\infty} a_{n}, a_{n} \geq 0$, is convergent to some element $s$ if and only if the sequence $\left(s_{n}\right)_{n}(O)$-converges to $s$.

We now introduce a first concept of $\sigma$-additivity, similar to the classical one. (In the sequel we will slightly sharpen this concept.) 
Definition 2.4. Let $R$ be a Dedekind complete $l$-group, $\mathcal{F}$ be an algebra of subsets of a nonempty set $X$ and $m: \mathcal{F} \rightarrow R_{0}^{+}$be a finitely additive measure. We say that $m$ is order $\sigma$-additive if $m\left(\bigcup_{n=1}^{\infty} H_{n}\right)=\sum_{n=1}^{\infty} m\left(H_{n}\right)$ whenever $\left(H_{n}\right)_{n}$ is a disjoint sequence of elements of $\mathcal{F}$, such that $\bigcup_{n=1}^{\infty} H_{n} \in \mathcal{F}$.

In the sequel we shall assume further properties in our $l$-group, so we introduce now some definitions.

Definitions 2.5. A bounded double sequence $\left(a_{i, j}\right)_{i, j}$ in $R$, such that $a_{i, j} \downarrow 0$ for each $i \in \mathbb{N}$, is called a regulator or $(D)$-sequence.

For every $(D)$-sequence $\left(a_{i, j}\right)_{i, j}$, and every mapping $\phi: \mathbb{N} \rightarrow \mathbb{N}$, the element $\bigvee_{i=1}^{\infty} a_{i, \phi(i)}$ is called a domination of the $(D)$-sequence.

From now on, we shall denote by $\Phi$ the set of all mappings $\phi: \mathbb{N} \rightarrow \mathbb{N}$.

Now we can introduce the conditions we shall impose on $R$.

Definitions 2.6. We say that $R$ is weakly $\sigma$-distributive if, for every $(D)$ sequence $\left(a_{i, j}\right)_{i, j}$, the greatest lower bound of its dominations is 0 ; i.e.,

$$
\bigwedge_{\phi \in \Phi}\left(\bigvee_{i=1}^{\infty} a_{i, \phi(i)}\right)=0 .
$$

A Dedekind complete $l$-group $R$ is said to be super Dedekind complete, if for any nonempty set $A \subset R$, bounded from above, there exists a countable subset $A^{*} \subset A$, such that $\sup A=\sup A^{*}$.

From now on, we shall always assume that $R$ is a super Dedekind complete and weakly $\sigma$-distributive $l$-group.

The following lemma is a version of the Fremlin Lemma in the context of $(O)$-sequences. Though it is possible to prove it as a consequence of the Fremlin-type [6, Theorem 3.2.3, page 42], we give here a direct proof, because it looks somewhat easier.

Lemma 2.7. Let $\left(r_{n}\right)_{n}$ be any $(O)$-sequence in $R_{0}^{+}$. For every $U \in R_{0}^{+}$there exists an element $\omega \in \Phi$ such that the mapping $N \mapsto U \wedge \sum_{n=N}^{\infty} r_{\omega(n)}$ is an (O)-sequence.

Proof. For any couple $(i, k)$ of positive integers, set $A_{i, k}=U \wedge\left(\sum_{n=k}^{k+2^{i}-1} r_{n}\right)$. Clearly, $\left(A_{i, k}\right)_{i, k}$ is a $(D)$-sequence. Next, for every element $\phi \in \Phi$, define $d_{\phi}:=\bigvee_{i=1}^{\infty} A_{i, \phi(i)}$. Since $R$ is weakly $\sigma$-distributive and super Dedekind complete, there exists a sequence $\left(\phi_{h}\right)_{h}$ in $\Phi$, such that $\inf _{h} d_{\phi_{h}}=0$. Without loss of generality, we shall assume that $\phi_{h}(n)<\phi_{h}(n+1)$ and $\phi_{h}(n)<\phi_{h+1}(n)$ for every $h$ and $n$ in $\mathbb{N}$, so that $N \mapsto g_{N}:=d_{\phi_{N}}$ defines an $(O)$-sequence. Thus 
we have $g_{1} \geq U \wedge\left\{r_{\phi_{1}(1)}+r_{\phi_{1}(1)+1}\right\}$ and also $g_{1} \geq U \wedge\left\{r_{\phi_{1}(2)}+r_{\phi_{1}(2)+1}+\right.$ $\left.r_{\phi_{1}(2)+2}+r_{\phi_{1}(2)+3}\right\}$, and so on. Now we observe that

$$
\begin{gathered}
U \wedge 2 r_{\phi_{1}(1)+1} \leq g_{1}, U \wedge\left(r_{\phi_{1}(1)+1}+2 r_{\phi_{1}(2)+3}\right) \leq g_{1}, \\
U \wedge\left(r_{\phi_{1}(1)+1}+r_{\phi_{1}(2)+3}+2 r_{\phi_{1}(3)+7}\right) \leq g_{1}
\end{gathered}
$$

and so on. Then $U \wedge\left(\sum_{n=1}^{k} r_{\phi_{1}(n)+2^{n}-1}\right) \leq g_{1}$ holds for every positive integer $k$; hence $U \wedge\left(\sum_{n=1}^{\infty} r_{\phi_{1}(n)+2^{n}-1}\right) \leq g_{1}$. In a similar way, one proves that $U \wedge\left(\sum_{n=1}^{\infty} r_{\phi_{N}(n)+2^{n}-1}\right) \leq g_{N}$ holds, for every positive integer $N$. Now, we set $\omega(N):=\phi_{N}(N)+2^{N}-1$. For every natural number $k$, we have

$$
\begin{aligned}
U \wedge\left(\sum_{n=N}^{N+k} r_{\omega(n)}\right) & =U \wedge\left(\sum_{n=N}^{N+k} r_{\phi_{n}(n)+2^{n}-1}\right) \leq U \wedge\left(\sum_{n=N}^{N+k} r_{\phi_{N}(n)+2^{n}-1}\right) \\
& \leq U \wedge\left(\sum_{n=1}^{\infty} r_{\phi_{N}(n)+2^{n}-1}\right) \leq g_{N} .
\end{aligned}
$$

From the arbitrariness of $k$, we obtain the assertion.

The next result expresses the fact that, as soon as $\left\{\left(r_{n}^{(k)}\right)_{n}: k \in \mathbb{N}\right\}$ is an equibounded countable family of $(O)$-sequences, there exists a single $(O)$ sequence, which can replace them all. More precisely, we have the following.

Lemma 2.8. Let $\left\{\left(r_{n}^{(k)}\right)_{n}: k \in \mathbb{N}\right\}$ be an equibounded countable family of $(O)$ sequences. Then there exists an $(O)$-sequence $\left(b_{j}\right)_{j}$ with the following property: For every $j, k \in \mathbb{N}$, an integer $n=n(j, k)>0$ exists, such that $r_{n}^{(k)} \leq b_{j}$.

Proof. Define $a_{k, n}=r_{n}^{(k)}$ for each $k, n$. Clearly, $\left(a_{k, n}\right)_{k, n}$ is a $D$-sequence, hence there exists a sequence $\left(\phi_{j}\right)_{j}$ in $\Phi$, such that $j \mapsto b_{j}:=\bigvee_{n=1}^{\infty} a_{n, \phi_{j}(n)}$ defines an $(O)$-sequence. As above, we can assume that $\phi_{j}(n)<\phi_{j}(n+1)$ and $\phi_{j}(n)<\phi_{j+1}(n)$ for all $j, n$. Arbitrarily fix $j$ and $k$, and choose $n=$ $n(j, k)=\phi_{j}(k)$. We have then $r_{n}^{(k)}=a_{k, \phi_{j}(k)} \leq \bigvee_{k=1}^{\infty} a_{k, \phi_{j}(k)}=b_{j}$, which is the assertion.

From now on, we denote by $\mathcal{F}$ an algebra and by $\mathcal{A}$ a $\sigma$-algebra of subsets of a nonempty arbitrary set $X$.

We now introduce the concept of $s$-boundedness in the context of $(O)$ convergence.

Definition 2.9. A finitely additive measure $m: \mathcal{F} \rightarrow R$ is said to be $s$-bounded if there exists an $(O)$-sequence $\left(b_{j}\right)_{j}$ such that, for every disjoint sequence $\left(H_{k}\right)_{k}$ in $\mathcal{F}$ and every index $j \in \mathbb{N}$, one can find an integer $k_{0}$ satisfying

$$
\left|m\left(H_{k}\right)\right| \leq b_{j} \text { for each } k \in \mathbb{N}, k \geq k_{0} .
$$


We say that the measures $m_{n}: \mathcal{F} \rightarrow R, n \in \mathbb{N}$, are uniformly s-bounded, if the integer $k_{0}$ in (1) can be chosen independently of $n$.

We now introduce the notion of $\sigma$-additivity in the context of $(O)$-convergence. This concept is, in general, stronger than the classical one of order $\sigma$-additivity.

Definition 2.10. Let $m: \mathcal{F} \rightarrow R$ be a finitely additive measure. We say that $m$ is $\sigma$-additive if there exists an $(O)$-sequence $\left(b_{j}\right)_{j}$ such that, for every sequence $\left(H_{k}\right)_{k}$ in $\mathcal{F}$, decreasing to the empty set, and for every positive integer $j$, there exists a natural number $k_{0}$ satisfying $|m(B)| \leq b_{j}$ for every $B \in \mathcal{F}, B \subset H_{k_{0}}$. A similar definition concerns uniform $\sigma$-additivity for a family $\left\{m_{i}: \mathcal{F} \rightarrow R\right\}_{i}$ of measures.

We observe that, in case of an equibounded sequence $\left(m_{n}\right)_{n}$ of $\sigma$-additive measures, it is possible to find a unique $(O)$-sequence $\left(b_{j}\right)_{j}$ which is related to the $\sigma$-additivity of all the $m_{n}$. This is a consequence of Lemma 2.8. The same also holds for other properties,such as continuity, to be introduced later.

From the extension theorems found in [4], it is possible to deduce that any $s$-bounded positive order $\sigma$-additive measure is also $\sigma$-additive.

Some of these extension theorems will now be recalled, for completeness, and also for further reference.

We first introduce some notation: given an algebra $\mathcal{F}$ of subsets of any nonempty set $X$, we denote by $\sigma(\mathcal{F})$ the $\sigma$-algebra generated by $\mathcal{F}$.

A stronger notion of convergence is also needed.

Definition 2.11. Let $T$ be any nonempty set, and $\left(f_{n}: T \rightarrow R\right)_{n}$ be any sequence of functions. We say that the sequence $\left(f_{n}\right)_{n}$ is $(R O)$-convergent to a limit function $f$ if there exists an $(O)$-sequence $\left(p_{j}\right)_{j}$ in $R$ such that, for every positive integer $j$ and every element $t \in T$, a natural number $n_{0}$ can be found, $n_{0}=n_{0}(j, t)$, for which $\left|f_{n}(t)-f(t)\right| \leq p_{j} \quad \forall n \geq n_{0}$.

This definition will be mainly used for sequences of $R$-valued measures.

The theorems we list deal mainly with the so-called Stone Isomorphism technique. The well-known Stone Isomorphism Theorem asserts that any Boolean algebra $\mathcal{F}$ is algebraically isomorphic with the algebra $\Sigma$ of clopen sets in a suitable compact, totally disconnected, Hausdorff space $S$. Denoting by $\psi: \mathcal{F} \rightarrow \Sigma$ such an isomorphism, any finitely additive measure $m: \mathcal{F} \rightarrow R$ can be associated with the measure $m \circ \psi^{-1}: \Sigma \rightarrow R$. Since $\Sigma$ turns out to be perfect, any finitely additive measure on $\Sigma$ is also order $\sigma$-additive.

Thus a suitable extension procedure, inspired by Carathéodory's construction, yields the following theorem [4]. 
Theorem 2.12. Let $m: \mathcal{F} \rightarrow R_{0}^{+}$be any finitely additive, $s$-bounded measure. There exists a $\sigma$-additive measure $\widetilde{m}: \sigma(\Sigma) \rightarrow R_{0}^{+}$such that $\widetilde{m}_{\mid \Sigma}=m \circ \psi^{-1}$. Moreover, there exists a suitable $(O)$-sequence $\left(b_{j}\right)_{j}$ in $R$, such that, for every element $A \in \sigma(\Sigma)$ and each positive integer $j$, an element $F \in \mathcal{F}$ can be found, satisfying $\widetilde{m}(A \Delta \psi(F)) \leq b_{j}$.

Combining this theorem with a convergence theorem of the type of VitaliHahn-Saks [3, 4], the following result can be deduced.

Theorem 2.13. Let $\left(m_{n}: \mathcal{A} \rightarrow R\right)_{n}$ be an equibounded sequence of $s$-bounded finitely additive measures, defined on a $\sigma$-algebra $\mathcal{A}$. Assuming that the sequence $\left(m_{n}(A)\right)_{n}$ is $(R O)$-convergent to a limit $m(A), \forall A \in \mathcal{A}$, then: (i) the sequence $\left(m_{n}\right)_{n}$ is uniformly s-bounded, and therefore $m$ is s-bounded too; and, (ii) the sequence $\left(\widetilde{m_{n}}(B)\right)_{n}$ is $(R O)$-convergent to $\widetilde{m}(B), \forall B \in \sigma(\Sigma)$, where as usual $\Sigma$ denotes the Stone algebra isomorphic with $\mathcal{A}$, and $\widetilde{m_{n}}, \widetilde{m}$ are the Stone extensions to the $\sigma$-algebra $\sigma(\Sigma)$ of $m_{n}$ and $m$ respectively.

\section{Continuous and Atomic Measures.}

We now introduce the concept of continuity for $l$-group-valued measures $[1,7]$.

Definition 3.1. We say that a finitely additive measure $m: \mathcal{F} \rightarrow R_{0}^{+}$is continuous if $\inf _{P \in \Pi}\left[\sup _{D \in P} m(D)\right]=0$, where $P$ is any finite partition of $X$, and the infimum is taken with respect to the totality $\Pi$ of such partitions. The finitely additive measures $m_{n}: \mathcal{F} \rightarrow R_{0}^{+}, n \in \mathbb{N}$, are said to be uniformly continuous if $\inf _{P \in \Pi}\left[\sup _{D \in P}\left(\sup _{n} m_{n}(D)\right)\right]=0$.

The following result is a characterization of continuity for finitely additive positive measures.

Proposition 3.2. A finitely additive measure $m: \mathcal{F} \rightarrow R_{0}^{+}$is continuous if and only if there exists an $(O)$-sequence $\left(b_{j}\right)_{j}$ in $R$ such that for all $j \in \mathbb{N}$ there exists a finite partition $Q_{j}$ of $X$ into sets $D_{1}, \ldots, D_{h_{j}}$, for which

$$
m\left(D_{i}\right) \leq b_{j} \quad \forall i=1, \ldots, h_{j} .
$$

Analogously, the finitely additive measures $m_{n}: \mathcal{F} \rightarrow R_{0}^{+}, n \in \mathbb{N}$, are uniformly continuous if, and only if, there exists an $(O)$-sequence $\left(b_{j}\right)_{j}$ in $R$ such that for all $j \in \mathbb{N}$, there exists a finite partition $Q_{j}$ of $X$ into sets $D_{1}, \ldots, D_{h_{j}}$, satisfying $\sup _{n} m_{n}\left(D_{i}\right) \leq b_{j} \quad \forall i=1, \ldots, h_{j}$.

Proof. We give the proof only for the first part of the proposition. Let $m$ be a positive continuous finitely additive measure. Since $R$ is super Dedekind complete, then there exists a sequence $\left(Q_{j}\right)_{j}$ of finite partitions of $X$, such 
that $\inf _{j}\left[\sup _{D \in Q_{j}} m(D)\right]=0$. Without loss of generality, we can assume that the sequence $\left(Q_{j}\right)_{j}$ is increasing with respect to the refinement order; i.e., we assume that, for every positive integer $j$, each element in $Q_{j}$ is the union of some elements from $Q_{j+1}$.

For every index $j \in \mathbb{N}$, define $b_{j}:=\sup _{D \in Q_{j}} m(D)$. Thus, $\left(b_{j}\right)_{j}$ turns out to be an $(O)$-sequence, and the sequence $\left(Q_{j}\right)_{j}$ is the required one.

Conversely, let $\left(Q_{j}\right)_{j}$ be a sequence of finite partitions of $X$, satisfying (2). We get $\inf _{j}\left[\sup _{D \in Q_{j}} m(D)\right]=0$ and, a fortiori, $\inf _{P \in \Pi}\left[\sup _{D \in P} m(D)\right]=0$, that is, continuity of $m$.

Definition 3.3. A finitely additive measure $m: \mathcal{A} \rightarrow R_{0}^{+}$is said to be atomic if 0 is the unique finitely additive and continuous measure $\nu: \mathcal{A} \rightarrow R_{0}^{+}$, satisfying $\nu \leq m$.

We note that the given definition of atomic measure agrees with the classical one of atomic $\mathbb{R}_{0}^{+}$-valued measure, by virtue of the Sobczyk-Hammer decomposition theorem for scalar measures [7].

In the sequel, a suitable notion of absolute continuity is needed. We introduce it now, only for $\sigma$-additive measures, and will then deduce a useful result about continuity of measures.

Definition 3.4. Let $\nu: \mathcal{A} \rightarrow R_{0}^{+}$be any $\sigma$-additive measure, defined on a $\sigma$-algebra $\mathcal{A}$. Given any other $\sigma$-additive measure $m: \mathcal{A} \rightarrow R_{0}^{+}$, we say $m$ is absolutely continuous with respect to $\nu$ if $\nu(A)=0 \Rightarrow m(A)=0$.

Here is the theorem concerning continuity.

Theorem 3.5. Let $m, \nu: \mathcal{A} \rightarrow R_{0}^{+}$, and assume $m$ and $\nu$ are $\sigma$-additive, $m$ is absolutely continuous with respect to $\nu$ and $\nu$ is continuous. Then $m$ is continuous.

Proof. Set $U:=m(X)+\nu(X)$. Fix an $(O)$-sequence $\left(b_{j}\right)_{j}$ in $R$, agreeing with the $\sigma$-additivity of $m$, with $\sigma$-additivity and with continuity of $\nu$. Thanks to Lemma 2.7, there exists a subsequence $\left(b_{j_{k}}\right)_{k}$ such that $\left(\rho_{N}\right)_{N}$ is an $(O)$ sequence, where $\rho_{N}:=U \wedge \sum_{k>N} b_{j_{k}}$ for all $N$. Set $b_{N}^{\prime}:=b_{j_{N}}$, and define $\alpha_{N}:=\sup \left\{m(A): \nu(A) \leq b_{N}^{\prime}\right\}, \forall N \in \mathbb{N}$. We shall prove that $\left(\alpha_{N}\right)_{N}$ is an $(O)$-sequence. Clearly, $\left(\alpha_{N}\right)_{N}$ is decreasing, so all that must be shown is $\alpha:=\inf _{N} \alpha_{N}$ coincides with 0 .

If this is not the case, then there exists an integer $N$ such that $\alpha \not \leq b_{N}$; hence $\alpha_{k} \not \leq b_{N} \forall k$. This means that, for every natural number $k$, an element $A_{k} \in \mathcal{A}$ can be found, such that $\nu\left(A_{k}\right) \leq b_{k}^{\prime}$, but $m\left(A_{k}\right) \not \leq b_{N}$. For all positive integers $s$, define $A_{s}^{*}:=\bigcup_{k \geq s} A_{k}$. We get $\nu\left(A_{s}^{*}\right) \leq U \wedge \sum_{k \geq s} b_{k}^{\prime} \leq \rho_{s}$, but

$$
m\left(A_{s}^{*}\right) \not \leq b_{N},
$$


for all $s$. The sequence $\left(A_{s}^{*}\right)_{s}$ is decreasing. Denoting its limit by $A$, we get $\nu(A)=0$ by $\sigma$-additivity, and then $m(A)=0$. Hence, the sequence $\left(A_{s}^{*} \backslash A\right)_{s}$ is decreasing to $\emptyset$, and $m\left(A_{s}^{*} \backslash A\right)=m\left(A_{s}^{*}\right)$ for all $s$. Thus, by $\sigma$-additivity, a positive integer $\tau_{N}$ can be found, satisfying $m\left(A_{\tau_{N}}^{*}\right) \leq b_{N}$. But this is contrary to (3). We must conclude that $\alpha=0$.

Now, we can easily prove the continuity of $m$. Indeed, for each $N \in \mathbb{N}$, a partition $P$ of $X$ exists, such that $\nu(D) \leq b_{N}^{\prime}$ for all $D \in P$. Then $m(D) \leq \alpha_{N}$ for all $D \in P$, and the assertion is proved.

We now prove that, for continuous equibounded finitely additive measures, uniform $s$-boundedness is a sufficient condition for uniform continuity. To this aim, we begin with the following definition.

Definition 3.6. Let $m: \mathcal{F} \rightarrow R_{0}^{+}$be any set function, and fix $u \in R_{0}^{+}$. Given any set $A \in \mathcal{F}$, we say that $A$ is $u$-decomposable (with respect to $m$ ), if there exists a finite partition of $A$ into sets $D_{1}, \ldots, D_{k}$ of $\mathcal{F}$, such that $m\left(D_{i}\right) \leq u$ for all $i=1, \ldots, k$. Thus we get that a positive finitely additive measure $m$ is continuous if, and only if, there exists an $(O)$-sequence $\left(r_{j}\right)_{j}$ in $R$ such that, for each $j \in \mathbb{N}$, the set $X$ is $r_{j}$-decomposable (see also Proposition 3.2).

Let $\left(m_{n}: \mathcal{F} \rightarrow R_{0}^{+}\right)_{n}$ be a sequence of finitely additive equibounded measures. For every $A \in \mathcal{F}$, set $M(A):=\sup _{n \in \mathbb{N}} m_{n}(A)$. Given $A \in \mathcal{F}$, we say that $A$ is uniformly $u$-decomposable if it is $u$-decomposable with respect to $M$.

Thus we get that the $m_{n}$ 's are uniformly continuous if, and only if, there exists an $(O)$-sequence $\left(r_{j}\right)_{j}$ in $R$ such that, for each $j \in \mathbb{N}$, the set $X$ is uniformly $r_{j}$-decomposable.

We also need the following lemma.

Lemma 3.7. Let $\left(m_{n}: \mathcal{F} \rightarrow R_{0}^{+}\right)_{n}$ be a sequence of continuous, equibounded and uniformly s-bounded measures, and let $\left(r_{j}\right)_{j}$ be an $(O)$-sequence, according with the uniform s-boundedness and continuity of each measure $m_{n}$. Assume that, for some $j \in \mathbb{N}$, there exists $A \in \mathcal{F}$, with $M(A) \not \leq 2 r_{j}$. Then there exists $H \subset A, H \in \mathcal{F}$, uniformly $r_{j}$-decomposable and such that $M(H) \not \leq 2 r_{j}$.

Proof. Let $\left(r_{j}\right)_{j}$ be as in the hypotheses. We note that such a sequence does exist, by virtue of Lemma 2.8 and equiboundedness. Without loss of generality, suppose $j=1$, and set $r=r_{1}$. By contradiction, suppose that there exists $A \in \mathcal{F}$, with $M(A) \not \leq 2 r$, such that there are no uniformly $r$ decomposable subsets $H \in \mathcal{F}$, with $M(H) \not \leq 2 r$. Without loss of generality, suppose that $m_{1}(A) \not \leq 2 r$. By the continuity of $m_{1}$, there exists a partition $\mathcal{D}_{A}$ of $A$ into sets $D_{1}, \ldots, D_{k}$, such that $m_{1}\left(D_{i}\right) \leq r$ for all $i=1, \ldots, k$. By virtue of the assumed contradiction, we get $M\left(D_{k_{1}}\right) \not \leq 2 r$ for some index $k_{1} \leq k$, and thus, in correspondence with $k_{1}$, there exists an integer $n_{1}>1$ such that 
$m_{n_{1}}\left(D_{k_{1}}\right) \not \leq 2 r$. Set $A_{1}:=D_{k_{1}}$ and $B_{1}:=A \backslash A_{1}$. By difference, we get $m_{1}\left(B_{1}\right) \not \leq r$. By the continuity of $m_{1}, m_{2}, \ldots m_{n_{1}}$, there exists a partition $\mathcal{D}$ of $A_{1}$ into sets $D_{1}^{\prime}, D_{2}^{\prime}, \ldots, D_{s}^{\prime}$, such that $m_{1}\left(D_{i}^{\prime}\right) \vee m_{2}\left(D_{i}^{\prime}\right) \vee \ldots \vee m_{n_{1}}\left(D_{i}^{\prime}\right) \leq r$ for each $i=1, \ldots, s$. Again by contradiction, it follows: $M\left(D_{k_{2}}^{\prime}\right) \not \leq 2 r$ for a suitable index $k_{2} \leq s$, and hence there exists an integer $n_{2}>n_{1}$ such that $m_{n_{2}}\left(D_{k_{2}}^{\prime}\right) \not \leq 2 r$. Put $A_{2}:=D_{k_{2}}^{\prime}$, and $B_{2}:=A_{1} \backslash A_{2}$. By difference, we get $m_{n_{1}}\left(B_{2}\right) \not \leq r$. By virtue of continuity of $m_{1}, m_{2}, \ldots, m_{n_{2}}$, select a partition of $A_{2}$ and a set $A_{3} \subset A_{2}, A_{3} \in \mathcal{F}$, such that $M\left(A_{3}\right) \not \leq 2 r$ and $m_{n_{2}}\left(A_{3}\right) \leq r$. Setting $B_{3}:=A_{2} \backslash A_{3}$, by difference we have $m_{n_{2}}\left(B_{3}\right) \not \leq r$. Proceeding in this way, we find an increasing sequence of natural numbers $\left(n_{h}\right)_{h}$ and a decreasing sequence of sets $\left(A_{h}\right)_{h}$ in $\mathcal{F}$, such that $m_{n_{h}}\left(B_{h+1}\right) \not z r$ for all $h \in \mathbb{N}$, where $\left(B_{h}:=A_{h} \backslash A_{h+1}\right)_{h}$ is a disjoint sequence in $\mathcal{F}$. This contradicts uniform $s$-boundedness, and the lemma is proved.

We finally turn to the announced useful result.

Theorem 3.8. Under the same notations as in Lemma 3.7, if the $m_{n}$ 's are positive, continuous, equibounded and uniformly s-bounded finitely additive $R$ valued measures, then they are uniformly continuous.

Proof. By virtue of equiboundedness and thanks to Lemma 2.8, there exists an $(O)$-sequence $\left(r_{j}\right)_{j}$, agreeing both with the uniform $s$-boundedness and with the continuity of each measure $m_{n}$. To prove the theorem, we shall show that, for every $j \in \mathbb{N}, X$ is uniformly $2 r_{j}$-decomposable. Fix any positive integer $j$, and set $r=r_{j}$. Suppose, for purposes of contradiction, that $X$ is not uniformly $2 r$-decomposable. (From now on in this proof, the term "decomposable" will always mean "uniformly decomposable.") Then $M(X) \not \leq 2 r$, and thus, by Lemma 3.7, there exists a set $A_{1} \in \mathcal{F}$, with $M\left(A_{1}\right) \not \leq 2 r$, but $r$-decomposable. By the assumed contradiction, $X \backslash A_{1}$ is not $2 r$-decomposable, and hence we have $M\left(X \backslash A_{1}\right) \not \leq 2 r$. Again by Lemma 3.7, there exists an $r$-decomposable set $A_{2} \subset X \backslash A_{1}$, with $A_{2} \in \mathcal{F}$, such that $M\left(A_{2}\right) \not \leq 2 r$. Since $A_{1}$ and $A_{2}$ are $r$-decomposable and $X$ is not, then $X \backslash\left(A_{1} \cup A_{2}\right)$ is not $2 r$-decomposable, and thus, again by Lemma 3.7, there exists an $r$-decomposable set $A_{3}$ in $\mathcal{F}$, disjoint both from $A_{1}$ and from $A_{2}$, such that $M\left(A_{3}\right) \not \leq 2 r$. Thus we get the existence of a sequence $\left(A_{k}\right)_{k}$ of pairwise disjoint $r$-decomposable sets of $\mathcal{F}$, such that $M\left(A_{k}\right) \not 22 r, \forall k \in \mathbb{N}$. This contradicts uniform $s$-boundedness of the measures $m_{n}$, and thus $X$ is $2 r$-decomposable. So the assertion follows.

\section{Sobczyk-Hammer Decompositions.}

In this section, we deduce existence and convergence theorems for SobczykHammer decompositions, first for $\sigma$-additive measures, and then for finitely additive ones. 
For $\sigma$-additive measures $m$, we shall obtain a decomposition of sectional type; i.e., we shall find a suitable set $H \in \mathcal{A}$, such that the restrictions of $m$ to $H$ and to $H^{c}$ are continuous and atomic respectively.

We begin with a lemma.

Lemma 4.1. Suppose $m: \mathcal{A} \rightarrow R$ is a positive, $\sigma$-additive measure. If $m$ is not atomic, then there exists at least a set $F \in \mathcal{A}$ with $m(F) \neq 0$, such that $m_{\mid F}$ is continuous.

Proof. Since $m$ is not atomic, there exists a continuous non-trivial measure $\mu: \mathcal{A} \rightarrow R_{0}^{+}$, such that $\mu \leq m$. It is readily seen that $\mu$ is $\sigma$-additive. Let now $\mathcal{H}$ be the family of all sets $H \in \mathcal{A}$ such that $\mu(H)=0$, and define

$$
\alpha=\sup \{m(H): H \in \mathcal{H}\} .
$$

By super Dedekind completeness, there exists a sequence $\left(K_{n}\right)_{n}$ in $\mathcal{H}$, such that $\alpha=\sup _{n} m\left(K_{n}\right)$. Without loss of generality, we can assume that the sequence $\left(K_{n}\right)_{n}$ is non-decreasing. Then, $m(K)=\alpha$ and $\mu(K)=0$, where $K=\bigcup_{n=1}^{\infty} K_{n}$.

Put $F=K^{c}$, and let us show that $F$ is the required set. First of all, it is easy to check that the measure $m_{\mid F}$ is absolutely continuous with respect to $\mu$. Indeed, if there exists a set $G \in \mathcal{A}$ such that $\mu(G)=0$ and $m_{\mid F}(G)>0$, then $m[(G \cap F) \cup K]=m(G \cap F)+m(K)>\alpha$. But $\mu[(G \cap F) \cup K]=$ $\mu(G \cap F)+\mu(K)=0$, and therefore $\alpha$ could not be the supremum in (4), a contradiction. Thus, by virtue of Theorem $3.5, m_{\mid F}$ is continuous. Finally we observe that $m(F) \neq 0$, otherwise $\mu \equiv 0$, which is impossible.

A useful consequence is the following.

Proposition 4.2. Let $m_{1}$ and $m_{2}$ be two atomic (continuous) $\sigma$-additive positive $R$-valued measures, defined on $\mathcal{A}$. Then $m_{1}+m_{2}$ is atomic (continuous).

Proof. The assertion concerning continuous measures is easy, so we deal only with the atomic case. Let us assume that $m_{1}$ and $m_{2}$ are atomic, and $m_{1}+m_{2}$ is not. Then there exists a set $F$, according with the previous Lemma. Thus $m_{1 \mid F}$ is continuous, and hence null. Similarly, $m_{2 \mid F}$ is null; hence we obtain $\left(m_{1}+m_{2}\right)_{\mid F}=0$, a contradiction.

We now turn to the following Sobczyk-Hammer-type theorem.

Theorem 4.3. If $m: \mathcal{A} \rightarrow R$ is a $\sigma$-additive positive measure, then there exists a set $E \in \mathcal{A}$, such that $m_{\mid E}$ is continuous and $m_{\mid E^{c}}$ is atomic. 
Proof. If $m$ is atomic, it is enough to take $E=\emptyset$. Otherwise, by virtue of Lemma 4.1, there exists $F \in \mathcal{A}$ such that $m(F) \neq 0$ and $m_{\mid F}$ is continuous. Denote by $\mathcal{K}$ the family of such sets, and write $\alpha:=\sup \{m(F): F \in \mathcal{K}\}$. By super Dedekind completeness of $R$, there exists an increasing sequence $\left(F_{n}\right)_{n}$ in $\mathcal{K}$, such that $\sup _{n} m\left(F_{n}\right)=\alpha$. Put $E=\bigcup_{n=1}^{\infty} F_{n}$. We prove that $E$ is the requested set. First of all, note that

$$
m_{\mid E}(A)=\sup _{n} m_{\mid F_{n}}(A)
$$

for all $A \in \mathcal{A}$. Moreover, it is easy to check that the $m_{\mid F_{n}}$ 's, $n \in \mathbb{N}$, are uniformly $s$-bounded. By Theorem 3.8, these measures are uniformly continuous, and hence, by (5), $m_{\mid E}$ is $\sigma$-additive and continuous.

Finally we prove that $m_{\mid E^{c}}$ is atomic. Otherwise, by Theorem 4.1, there exists a set $H \in \mathcal{A}$, such that $m(H) \neq 0, H \cap E=\emptyset$ and $m_{\mid H}$ is continuous. Then $H \cup E \in \mathcal{K}$ and $m(H \cup E)>\alpha$, a contradiction.

We now prove some convergence theorems for Sobczyk-Hammer-type decompositions.

First, consider the case of positive, $\sigma$-additive, $(R O)$-convergent measures. Recall that a sequence $\left(m_{k}\right)_{k}$ of finitely additive measures, defined on an algebra $\mathcal{F}$ with values in $R$, is (RO)-convergent to a measure $m$, if there exists an $(O)$-sequence $\left(r_{n}\right)_{n}$ such that, for all $A \in \mathcal{F}$ and $n \in \mathbb{N}$, an integer $k_{0}$ can be found, such that $\left|m_{k}(A)-m(A)\right| \leq r_{n}$ for all $k \geq k_{0}$.

In order to give convergence theorems for decompositions, we first prove the following.

Proposition 4.4. Let $\left(m_{n}\right)_{n}$ be a sequence of $\sigma$-additive positive $R$-valued measures, defined on a $\sigma$-algebra $\mathcal{A}$. If the $m_{n}$ 's are equibounded, then there exists $H \in \mathcal{A}$ such that $m_{n \mid H}$ is continuous and $m_{n \mid H^{c}}$ is atomic for every $n \in \mathbb{N}$.

Proof. For any $N \in \mathbb{N}$, denote by $\mu_{N}$ the measure $\mu_{N}:=\sum_{i=1}^{N} m_{i}$, and let $\left(A_{N}, A_{N}^{c}\right.$ ) be a Sobczyk-Hammer sectional decomposition of $\mu_{N}$ (See Theorem 4.3.). Set now, for positive integers $N, L$, with $N \leq L: B_{N, L}:=\bigcup_{p=0}^{L-N} A_{N+p}$, $B_{N}:=\bigcup_{j=N}^{\infty} B_{N, j}=\bigcup_{j=N}^{\infty} A_{j}$. We have

$$
m_{N \mid B_{N, L}} \leq \sum_{p=0}^{L-N}\left(\mu_{N+p}\right)_{\mid A_{N+p}}
$$

and hence $m_{N \mid B_{N, L}}$ is continuous. Letting $L \rightarrow \infty$, we deduce, by uniform $s$-boundedness, that the measure $m_{N \mid B_{N}}$ is continuous (see Theorem 3.8). 
Clearly, $\mu_{N \mid B_{N}^{c}} \leq \mu_{N \mid A_{N}^{c}}$ is atomic, for every $N$. Set now

$$
H:=\bigcap_{N=1}^{\infty} B_{N}
$$

We shall show that $H$ is the requested set. Indeed, for every $N \in \mathbb{N}$, we have $m_{N \mid H} \leq m_{N \mid B_{N}}$; hence $m_{N \mid H}$ is continuous.

We finally prove that $m_{N \mid H^{c}}$ is atomic, for all $N$. To this aim, fix any integer $N \in \mathbb{N}$, and let $\beta$ be any positive measure, $\beta \leq m_{N \mid H^{c}}$ : we deduce easily that $\beta_{\mid B_{N+p}^{c}} \leq m_{N \mid B_{N+p}^{c}} \leq\left(\mu_{N+p}\right)_{\mid B_{N+p}^{c}}$ for all $p \in \mathbb{N}$, and hence $\beta_{\mid B_{N+p}^{c}}$ is null, by atomicity of $\left(\mu_{N+p}\right)_{\mid B_{N+p}^{c}}$. But $\beta_{\mid H^{c}}=(R O) \lim _{p \rightarrow \infty} \beta_{\mid B_{N+p}^{c}}$; hence $\beta$ is null. This concludes the proof, by arbitrariness of $N$.

We now prove a first convergence theorem for Sobczyk-Hammer-type decompositions.

Theorem 4.5. Let $\left(m_{n}\right)_{n}$ be a sequence of $\sigma$-additive positive $R$-valued measures, defined on a $\sigma$-algebra $\mathcal{A}$. Suppose the $m_{n}$ are equibounded and $(R O)$ convergent to a measure $m$. Then $m$ is $\sigma$-additive, and the sequences $\left(m_{n}^{1}\right)_{n}$ and $\left(m_{n}^{2}\right)_{n}$ are $(R O)$-convergent to the measures $m^{1}$ and $m^{2}$, where $\left(m_{n}^{1}, m_{n}^{2}\right)$, $\left(m^{1}, m^{2}\right)$ are the sectional Sobczyk-Hammer decompositions of $m_{n}$ and $m$ respectively, $n \in \mathbb{N}$.

Proof. By virtue of the Vitali-Hahn-Saks theorem [4], the measures $m_{n}$ are uniformly $s$-bounded, and hence the limit measure $m$ is $\sigma$-additive. By applying Proposition 4.4 to the sequence $\left(m_{n}\right)_{n}$, we get the existence of a set $H \in \mathcal{A}$, which yields a sectional decomposition of the measures $m_{n}, n \in \mathbb{N}$, and of the limit measure $m$. The assertion follows immediately from $(R O)$ convergence.

We now turn to the finitely additive case.

Theorem 4.6. Let $m: \mathcal{F} \rightarrow R$ be any positive, finitely additive, s-bounded measure, defined on an algebra $\mathcal{F}$. There exists a decomposition $m=m^{1}+m^{2}$ of $m$ into two positive, finitely additive measures, such that $m^{1}$ is continuous and $m^{2}$ is atomic.

Proof. We make use of the Stone isomorphism; namely we consider the Stone space $S$ associated with $\mathcal{F}$, and the algebraic isomorphism $\psi$ from $\mathcal{F}$ to the algebra $\Sigma$ of all clopen subsets of $S$. From Theorem 2.12, the measure $m^{0}:=$ $m \circ \psi^{-1}$ can be extended to a $\sigma$-additive measure $\widetilde{m}$ on $\sigma(\Sigma)$. Using Theorem 4.4 , decompose $\widetilde{m}$ into the sum $\widetilde{m}^{1}+\widetilde{m}^{2}$, in the sense of Sobczyk-Hammer, 
where $\widetilde{m}^{1}$ is continuous and $\widetilde{m}^{2}$ is atomic. Now, restrict $\widetilde{m}^{1}$ and $\widetilde{m}^{2}$ to the algebra $\Sigma$, thus obtaining two measures, denoted by $m_{S}^{1}$ and $m_{S}^{2}$ respectively.

We shall see that $m_{S}^{1}$ and $m_{S}^{2}$ are continuous and atomic, respectively. From this, it will follow immediately that the measures, $m^{1}:=m_{S}^{1} \circ \psi$ and $m^{2}:=m_{S}^{2} \circ \psi$, give the requested decomposition of $m$. Let us prove that $m_{S}^{1}$ is continuous. By the continuity of $\widetilde{m}^{1}$, there exists an $(O)$-sequence $\left(a_{j}\right)_{j}$ such that, for all $j \in \mathbb{N}$, a finite partition $\left\{D_{1}, \ldots, D_{h_{j}}\right\}$ in $\sigma(\Sigma)$ can be found, satisfying $\widetilde{m}^{1}\left(D_{k}\right) \leq a_{j}, \forall k=1, \ldots, h_{j}$. Now, by virtue of Theorem 2.12, there exists an $(O)$-sequence $\left(b_{j}\right)_{j}$ such that, for all $D \in \sigma(\Sigma)$ and $j \in \mathbb{N}$, it is possible to find $E \in \Sigma$ such that $\widetilde{m}^{1}(E \Delta D) \leq b_{j}$.

Moreover, thanks to Lemma 2.7, and denoting by $U$ any majorant for all elements $m_{n}(X), n \in \mathbb{N}$, there exists a subsequence $\left(b_{j_{l}}\right)_{l}$ such that $N \mapsto$ $U \wedge \sum_{l=N}^{\infty} b_{j_{l}}$ is still an $(O)$-sequence. Let us call $\left(\rho_{N}\right)_{N}$ such a sequence.

The $(O)$-sequence $\left(a_{N}+\rho_{N}\right)_{N}$ can be used to prove continuity of $m_{S}^{1}$.

To this aim, fix any $N \in \mathbb{N}$. We can find a partition $\left\{D_{1}, \ldots, D_{h_{N}}\right\}$ in $\sigma(\Sigma)$, satisfying $\widetilde{m}^{1}\left(D_{k}\right) \leq a_{N}$ for all $k$. For each index $k$, from 1 to $h_{N}$, choose an element $E_{k} \in \Sigma$ such that $\widetilde{m}^{1}\left(E_{k} \Delta D_{k}\right) \leq b_{j_{(N+k)}}$. We have $\widetilde{m}^{1}\left(E_{k}\right) \leq a_{N}+\rho_{N}$, for all $k$.

Let $F_{1}=E_{1}, F_{2}=E_{2} \backslash E_{1}, \ldots, F_{h_{N}}=E_{h_{N}} \backslash\left(E_{1} \cup \ldots \cup E_{h_{N}-1}\right)$. Finally, set $F_{h_{N}+1}:=X \backslash \bigcup_{k=1}^{h_{N}} F_{k}=X \backslash \bigcup_{k=1}^{h_{N}} E_{k}$.

Of course, all the sets $F_{k}, k \in \mathbb{N}$, belong to $\Sigma$, and

$$
m_{S}^{1}\left(F_{k}\right)=\widetilde{m}^{1}\left(F_{k}\right) \leq b_{N}+\rho_{N} \forall k=1, \ldots, h_{N} .
$$

As to $F_{h_{N}+1}$, we see that

$$
\begin{aligned}
m_{S}^{1}\left(F_{h_{N}+1}\right) & =\widetilde{m}^{1}(X)-\sum_{k=1}^{h_{N}} \widetilde{m}^{1}\left(F_{k}\right)=\widetilde{m}^{1}\left(\bigcup_{k=1}^{h_{N}} D_{k} \backslash \bigcup_{k=1}^{h_{N}} E_{k}\right) \\
& \leq \widetilde{m}^{1}\left(\bigcup_{k=1}^{h_{N}}\left(D_{k} \Delta E_{k}\right)\right) \leq U \wedge \sum_{k=1}^{h_{N}} b_{j_{(N+k)}} \leq \rho_{N} .
\end{aligned}
$$

Hence, the partition $\left\{F_{1}, \ldots, F_{h_{N}}, F_{h_{N}+1}\right\}$ satisfies the condition $m_{S}^{1}\left(F_{k}\right) \leq$ $\rho_{N}+a_{N}$ for all $k=1, \ldots, h_{N}+1$, and thus $m_{S}^{1}$ is continuous.

We now prove the atomicity of $m_{S}^{2}$. Let $\nu: \Sigma \rightarrow R$ be a continuous positive finitely additive measure, such that $0 \leq \nu(A) \leq m_{S}^{2}(A), \forall A \in \Sigma$. Then $\nu$ admits a Carathéodory-type extension $\widetilde{\nu}$ to the whole of $\sigma(\Sigma)$. The continuity of $\widetilde{\nu}$ follows immediately from the continuity of $\nu$. Thus we get that $\widetilde{\nu}$ is a continuous finitely additive measure, such that $0 \leq \widetilde{\nu}(A) \leq \widetilde{m}^{2}(A), \forall A \in$ $\sigma(\Sigma)$. Thanks to the atomicity of $\widetilde{m}^{2}$, we get $\widetilde{\nu} \equiv 0$ on $\sigma(\Sigma)$, and thus, $a$ fortiori, $\nu \equiv 0$ on $\Sigma$. 
Before stating our final convergence theorem, we introduce some definitions, in order to also consider measures taking values not necessarily positive.

Definition 4.7. Let $\mathcal{F}$ be any algebra of subsets of a nonempty set $X$. Assume that $m: \mathcal{F} \rightarrow R$ is any finitely additive bounded measure. We put:

$$
\begin{aligned}
m^{+}(A) & =\sup \{m(B): B \in \mathcal{F}, B \subset A\}, \\
m^{-}(A) & =-\inf \{m(B): B \in \mathcal{F}, B \subset A\}, \\
v(m)(A) & =\sup \{|m(B)|: B \in \mathcal{F}, B \subset A\}
\end{aligned}
$$

for all $A \in \mathcal{F}$. The set functions $m^{+}, m^{-}, v(m)$ are called the positive variation, negative variation and semivariation of $m$ respectively. It is easy to see that $m^{+}$and $m^{-}$are positive finitely additive measures, $m^{+}-m^{-}=m$, and $v(m) \leq m^{+}+m^{-} \leq 2 v(m)$.

For any bounded finitely additive measure $m: \mathcal{F} \rightarrow R$, we shall say that $m$ is continuous (resp. atomic) if the measure $m^{+}+m^{-}$is.

From these definitions, it turns out immediately that any $s$-bounded finitely additive measure $m: \mathcal{F} \rightarrow R$ admits a Sobczyk-Hammer decomposition. It suffices to decompose $m^{+}$and $m^{-}$, and then apply Proposition 4.2.

We now state our final theorem.

Theorem 4.8. Let $\left(m_{n}\right)_{n}$ be any sequence of s-bounded, equibounded, finitely additive measures, defined on a $\sigma$-algebra $\mathcal{A}$ and taking values in $R$, and assume that $(R O) \lim _{n} m_{n}(A)=m(A)$ exists, for all $A \in \mathcal{A}$. Then, $m$ is $s$-bounded, and the sequences $\left(m_{n}^{1}\right)_{n}$ and $\left(m_{n}^{2}\right)_{n}$ are $(R O)$-convergent to the measures $m^{1}$ and $m^{2}$, where $\left(m_{n}^{1}, m_{n}^{2}\right),\left(m^{1}, m^{2}\right)$ are the continuous and the atomic parts which form the Sobczyk-Hammer decompositions of $m_{n}, n \in \mathbb{N}$ and $m$, respectively.

Proof. Again, we make use of the Stone isomorphism technique. Denote by $\Sigma$ the algebra of clopen sets, which is isomorphic to $\mathcal{A}$, and denote by $\psi: \mathcal{A} \rightarrow \Sigma$ such an isomorphism. Denote respectively by $\widetilde{m_{n}}, \widetilde{m_{n}^{+}}$, and so on, the countably additive extensions of $m_{n}, m_{n}^{+}$and so on, to the $\sigma$-algebra $\sigma(\Sigma)$. (We observe that $m_{n}, n \in \mathbb{N}$, and $m$ are $s$-bounded, and hence their Stone extensions, $\widetilde{m_{n}}, \widetilde{m}$ do exist.)

Thanks to Theorem 2.13, the sequence $\left(\widetilde{m_{n}}\right)_{n}$ is $(R O)$-convergent to $\widetilde{m}$ in $\sigma(\Sigma)$. Now, apply Proposition 4.4 to the sequences $\widetilde{\left(m_{n}^{+}\right)_{n}}$ and $\widetilde{\left(m_{n}^{-}\right)_{n}}$; thus obtaining a set $H \in \sigma(\Sigma)$ such that:

(1) $\left.\widetilde{m_{n}^{+}}\right|_{H}$ is continuous, $\left.\widetilde{m_{n}^{+}}\right|_{H^{c}}$ is atomic (and the same for $\widetilde{m_{n}^{-}}$);

(2) $\left.(R O) \lim _{n} \widetilde{m_{n}}\right|_{H}=\left.\widetilde{m}\right|_{H},\left.\quad(R O) \lim _{n} \widetilde{m_{n}}\right|_{H^{c}}=\left.\widetilde{m}\right|_{H^{c}}$. 
If we denote by $m_{n S}^{1}, m_{n S}^{2}, m^{1}{ }_{S}, m^{2}{ }_{S}$ the restrictions to $\Sigma$ of the measures $\left.\widetilde{m_{n}}\right|_{H},\left.\widetilde{m_{n}}\right|_{H^{c}},\left.\widetilde{m}\right|_{H},\left.\widetilde{m}\right|_{H^{c}}$, respectively, then $m_{n}^{1}:=m_{n S}^{1} \circ \psi, m_{n}^{2}:=m_{n S}^{2} \circ \psi$ are the Sobczyk-Hammer decompositions of the measures $m_{n}$, and $m^{1}:=m^{1}{ }_{S} \circ \psi$, $m^{2}:=m^{2}{ }_{S} \circ \psi$ give the Sobczyk-Hammer decomposition of $m$. Clearly, $(R O)$ convergence of the measures $\left.\widetilde{m_{n}}\right|_{H}$ and $\left.\widetilde{m_{n}}\right|_{H^{c}}$ implies $(R O)$-convergence of $m_{n}^{1}$ to $m^{1}$ and of $m_{n}^{2}$ to $m^{2}$.

\section{References}

[1] A. Boccuto, Continuous invariant vector-valued means and applications, Atti Sem. Mat. Fis. Univ. Modena, 43 (1995), 229-244.

[2] A. Boccuto, Egorov property and weak $\sigma$-distributivity in l-groups, Acta Math. (Nitra), 6 (2003) 61-66.

[3] A. Boccuto and D. Candeloro, Uniform s-boundedness and convergence results for measures with values in complete l-groups, J. Math. Anal. Appl., 265 (2002), 170-194.

[4] A. Boccuto and D. Candeloro, Convergence and decompositions for lgroup-valued set functions, Commentationes Math. Prace Mat., 44 (2003), 11-37.

[5] M. Duchoň and B. Riečan, On the Kurzweil-Stieltjes integral in ordered spaces, Tatra Mountains Math. Publ., 8 (1996), 133-141.

[6] B. Riečan and T. Neubrunn, Integral, Measure and Ordering (1997), Kluwer Acad. Publ./Ister Science, Dordrecht/Bratislava.

[7] A. Sobczyk and P. C. Hammer, A decomposition of additive set functions, Duke Math. J., 11 (1944), 839-846. 
